\title{
Factors affecting the corrosion fatigue life in nickel based superalloys for disc applications
}

\author{
Hollie Rosier ${ }^{1}$, a, Karen Perkins ${ }^{1}$, Andrew Girling ${ }^{1}$, Jonathan Leggett ${ }^{2}$, and Grant Gibson ${ }^{2}$ \\ ${ }^{1}$ College of Engineering, Swansea University, Singleton Park, Swansea, SA2 8PP, UK \\ 2 Rolls-Royce plc, PO Box 31, Derby, DE24 8BJ, UK
}

\begin{abstract}
The nickel based superalloy $720 \mathrm{Li}$ is employed in the gas turbine due to its mechanical performance at elevated temperature. A comprehensive assessment of the materials behaviour under representative service conditions is reported to address the drive for ever increasing temperatures and more arduous environmental exposure. Fatigue experiments have been performed in an air and air $/ \mathrm{SO}_{x}$ environment at $700{ }^{\circ} \mathrm{C}$ containing a mixed salt as a contaminant. There is an intimate relationship between local salt level (flux), stress level and stress state, i.e. static or cyclic. The interaction with these variables with the work hardened layer present on the surface of all tested specimens as a result of the shot peening process directly affects the crack initiation process. If specific conditions of environment and stress are achieved, a significant reduction in fatigue life is observed.
\end{abstract}

\section{Introduction}

Nickel based superalloys are widely employed within the gas turbine for their high temperature capability. In support of their application, extensive fatigue databases have been generated in the laboratory setting over a wide range of temperatures and mechanical conditions as a basis for either "life to first crack" or "damage tolerance" methodologies. A comprehensive, fundamental understanding of the initiation mechanisms and growth of fatigue cracks has been gained throughout the course of these previous studies.

Environmental contributions to this fatigue behaviour have long been assessed, particularly in the case of oxidation. For example, studies have employed high vacuum test facilities to partition environmental contributions from those of high temperature creepfatigue, whilst testing in atmospheric air at high temperatures can be employed to assess either the effects of prior or in situ exposure to oxidation. However, the drive to employ nickel alloys at ever increasing temperatures within the gas turbine may introduce a new form of environmental damage; that of high temperature corrosion. From the characterisation viewpoint, the simulation of such service conditions in the laboratory is extremely demanding and has restricted an alloys corrosion resistance being assessed under stress free conditions in the environment of interest. Any fatigue assessment or mechanical characterisation is then performed separately. However, recent evidence suggests that the separate assessment of such variables may not necessarily provide a true representation of the damage

\footnotetext{
${ }^{a}$ Corresponding author: $486639 @$ swansea.ac.uk
}

seen in service, where these conditions generally involve static or cyclic stress in combination with the corrosive atmosphere.

Under high temperature exposure, various authors have identified the propensity for Type II sulphidation in a number of different nickel based systems. Nicholls et al. [1] have investigated the controlling parameters behind such damage, in particular the roles played by surface condition, local in-situ gaseous chemistry and surface residues. Under such stress free conditions, damage usually proceeds via broad front attack and spallation of a relatively thick, stable, dual oxide layer consisting of a nickel oxide and mixed oxide ( $\mathrm{Ti}, \mathrm{Cr}, \mathrm{Al}$ ) with a sulphur rich band at the oxide metal interface. This type of attack generally results in wide shallow pitting and gross metal loss.

In contrast, evidence from components in service now suggest the addition of a mechanical stress cycle results in a new form of corrosion damage that results in narrower, deeper pitting. In this case, $\mathrm{Cr} / \mathrm{Ti}$ rich sulphides particles are seen decorating the grain boundaries ahead of the advancing corrosion front. Characterised as "early or stress enhanced" Type II hot corrosion the additional mechanical stress cycle, if of sufficient magnitude, results in localised rupture of the early dual oxide layer allowing diffusion of sulphur down grain boundaries resulting in localised grain boundary de-cohesion and grain drop out. This has implications from a fatigue point of view and full characterisation of the effect on mechanical performance is seen as an essential next step. The aim of the current work is to examine the key variables controlling the rate of pit formation and understand the resultant effect on mechanical properties such as fatigue life and crack propagation. 
Table 1. Chemical composition of $720 \mathrm{Li}$ wt. $\%$.

\begin{tabular}{|l|l|l|l|l|l|l|l|l|l|}
\hline Cr & Co & Mo & W & Al & Ti & C & B & Zr & Ni \\
\hline 16 & 15 & 3 & 1.25 & 2.5 & 5 & 0.015 & 0.015 & 0.035 & Bal \\
\hline
\end{tabular}

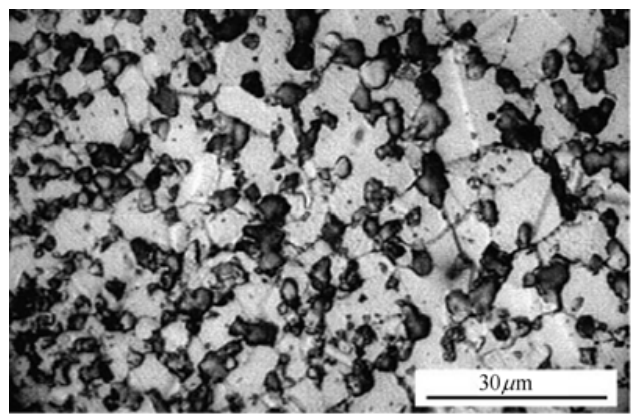

Figure 1. Optical micrograph of as-received 720Li revealed using Kalling's reagent [2].

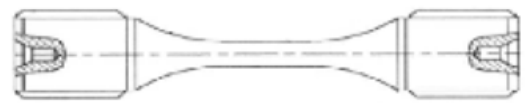

(a)

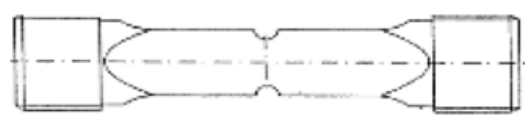

(b)

Figure 2. Schematic diagram of specimen design utilised, a) cylindrical design and b) notch specimen, $\mathrm{Kt}=2.2$.

\section{Material}

Experimental work and techniques developed throughout this study were conducted on the nickel superalloy, Udimet 720Li. 720Li has been developed for turbine disc applications due to its mechanical performance at elevated temperatures. The microstructure consists of a grain size of approximately $6-32 \mu \mathrm{m}$ with primary $\gamma^{\prime}\left(\mathrm{Ni}_{3}(\mathrm{Al}, \mathrm{Ti})\right)$ measuring 3-5 $\mu \mathrm{m}$ distributed on the grain boundaries, and secondary $\gamma$ ' measuring 70-120 nm, shown in Fig. 1 [2]. The chemical composition of $720 \mathrm{Li}$ is reported in Table 1.

Specimens were provided in the as machined, fully heat treated condition in the form of a double edge notch and cylindrical specimen design, Fig. 2. Specimens were in the shot peened condition.

\section{Experimental procedures}

Several key variables were identified that have the potential to affect corrosion mechanism, rate of damage formation and the mechanical properties of the alloy. The variables considered in this study are salt flux at a fixed $\mathrm{SO}_{2}$ content (ppm), stress amplitude/peak stress and waveform. A systematic study of each variable was performed as detailed in the following sections.

\subsection{Effect of salt flux}

The effect of salt flux on fatigue life was examined utilising the double edge notch specimen design. The salt solution
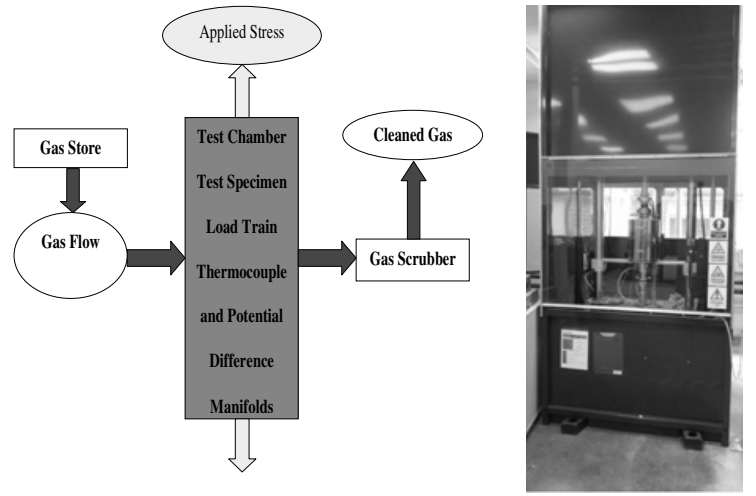

Figure 3. Schematic diagram of test setup for corrosion fatigue studies.

consisted of a fully saturated $98 \% \mathrm{Na}_{2} \mathrm{SO}_{4}-2 \% \mathrm{NaCl}$ mixture dissolved in a water/methanol mix. Full details of the development of this salting technique may be found elsewhere [3]. Specimens were pre-heated to a temperature of $120^{\circ} \mathrm{C}$ and utilising a micro-pipette, salt solution was added to each notch in $1 \mu \mathrm{l}$ batches until the desired level was reached. The use of a fully saturated solution prevented any re-dissolution of the already deposited salt and resulted in a uniform distribution of salt in the notch. Six fatigue tests were conducted in total covering a range of $5 \mu \mathrm{l}-50 \mu \mathrm{l}$.

Fatigue testing was performed at $700{ }^{\circ} \mathrm{C}$ within an environmental test chamber that encapsulated the load train to provide a continuous flow of an air/300ppm $\mathrm{SO}_{2}$ mix at $80 \mathrm{ccm}$. A schematic diagram of the test apparatus is shown in Fig. 3. Tests were conducted under load control utilising an, $\mathrm{R}=0$, trapezoidal waveform of 1-11-1 seconds at a single stress level. This value of stress corresponds to approximately mid stress/life range on an air $\mathrm{S} / \mathrm{N}$ curve for the same material. This also ensured failure within 100 hours, thus negating the need for specimen re-coating. This is desirable, as any interruption to the testing can significantly alter the final life of the test.

\subsection{Effect of stress}

The effect of stress on fatigue life was examined utilising a plain cylindrical specimen design. Prior to testing, salt was deposited on preheated specimens comprising a series of micropipette salt deposits of $4 \mu \mathrm{l}$ each positioned at 9 separate locations within the parallel gauge length of the specimen. This quantity of salt was identified from initial studies as that which gave comparable corrosion morphology as the previous specimen geometry at a similar level of stress. Any change in morphology/ mechanism during the tests would then be directly related to the change in stress and not from difference in test setup/specimen design.

Fatigue testing was again performed at $700{ }^{\circ} \mathrm{C}$ under a continuous flow of air/300vpm $\mathrm{SO}_{2}$ mix at $80 \mathrm{ccm}$. Tests were conducted under load control utilizing a fully reversed, $\mathrm{R}=-1$, trapezoidal waveform of 1-1-1-1 seconds at a range of peak stress to induce failure within a range of lifetimes from $10^{3}$ and $10^{5}$ cycles. Complete specimen rupture was selected as failure criteria. 


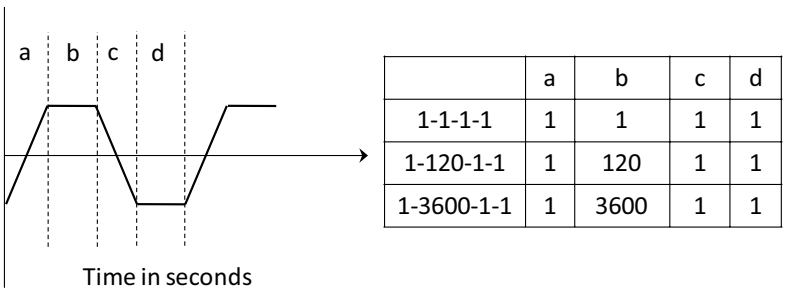

Figure 4. Schematic illustration of three waveforms utilised for examination of dwell effects.

\subsection{Effect of waveform}

The effect of waveform on fatigue life was examined utilising a plain cylindrical specimen design. Prior to testing, salt was deposited on preheated specimens comprising a series of micropipette salt deposits of $4 \mu \mathrm{l}$ each positioned at 9 separate locations within the parallel gauge length of the specimen as described previously.

Fatigue testing was again performed at $700^{\circ} \mathrm{C}$ under a continuous flow of air/300vpm $\mathrm{SO}_{2}$ mix at $80 \mathrm{ccm}$. Tests were conducted under load control, fully reversed, $\mathrm{R}=$ -1 . To allow for direct comparison with existing data and similar alloys, three standard trapezoidal waveforms were utilized (Fig. 4) at a range of peak stress to induce failure within a range of lifetimes from 10 and $10^{5}$ cycles. Complete specimen rupture was selected as failure criteria.

\subsection{Post test fractography and analysis}

Subsequent to testing, all failed specimens were inspected via optical and scanning electron microscopy (SEM/BSE and EDX) to identify the sites of fatigue crack initiation, specific fracture modes and detailed characterisation of corrosion/oxidation products.

\section{Results and discussion}

\subsection{Effect of salt flux}

The effect of salt flux on fatigue life is shown in Fig. 5, plotted as salt dosing per notch $(\mu \mathrm{l})$ versus number of cycles to failure. Air baseline and $\mathrm{SO}_{x}$ only data are also shown for comparison purposes. The data essentially describes a "C" shape whereby at the highest and lowest salt flux, the lives tend towards that obtained in Air or air/ $\mathrm{SO}_{x}$ only.

Detailed fractographic characterisation of the post test specimens indicate the data may be split into three separate regimes based on differences in failure mechanism.

Firstly, "high flux" or type II hot corrosion, demonstrates a thick dual oxide layer consisting nickel oxide and mixed oxide with a sulphur rich band between oxide and metal substrate, Fig. 6. This layer is typically $20-30 \mu \mathrm{m}$ thick. Surface damage or pitting is typically broad front in nature, i.e. wide shallow pitting. This type of morphology is typically observed during static corrosion testing and results in gross metal loss and spallation of

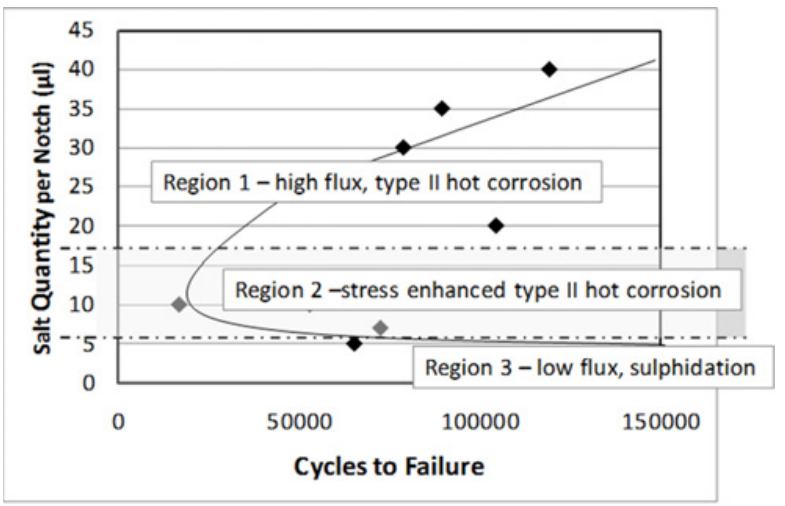

Figure 5. The effect of salt flux on fatigue life.
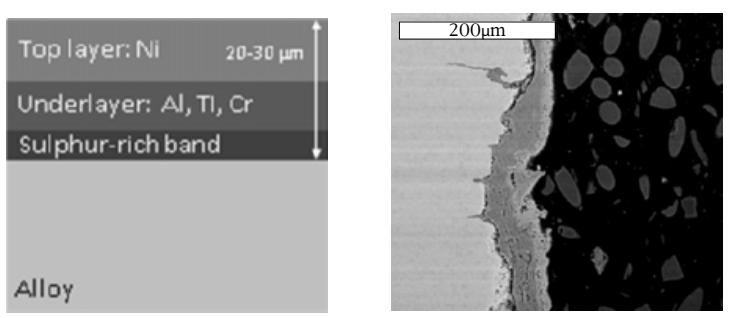

Figure 6. (a) Typical example of oxide developed under "high flux" or type II hot corrosion conditions.

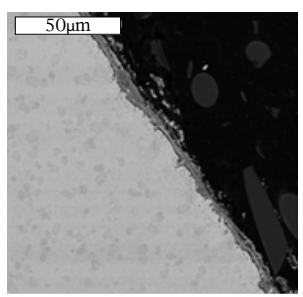

Figure 6. (b) Broad shallow pitting typical of high flux conditions. Backscattered electron image of polished surface (unetched) - principal stress axis parallel to oxide layer.
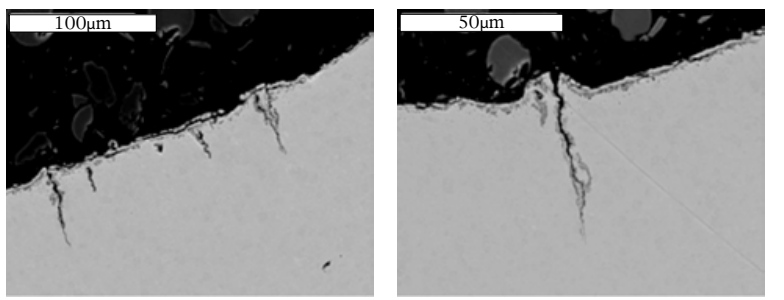

Figure 7. Typical example of sulphidation cracking found under low flux conditions. Backscattered electron image of polished surface (un-etched) - principal stress axis parallel to oxide layer.

the oxide layer [4]. Under fatigue testing, whilst causing a gradual reduction in section of the test specimens and corresponding increase in net section stress, it has minimal effect on overall life.

At "low flux" or sulphidation attack there simply is insufficient salt present to provide any significant attack Fig. 7. This type of cracking is indicative of $\mathrm{SO}_{x}$ only testing whereby oxide are much thinner, with characteristic "mounds" surrounding any surface cracking. Data tends towards that of air or air $/ \mathrm{SO}_{x}$ only. 


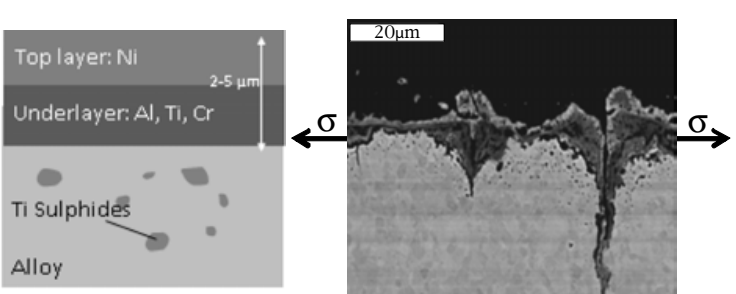

Figure 8. Typical example of oxide developed under "correct flux" or stress enhanced type II hot corrosion conditions. Backscattered electron image of polished surface (un-etched) principal stress axis parallel to oxide layer.

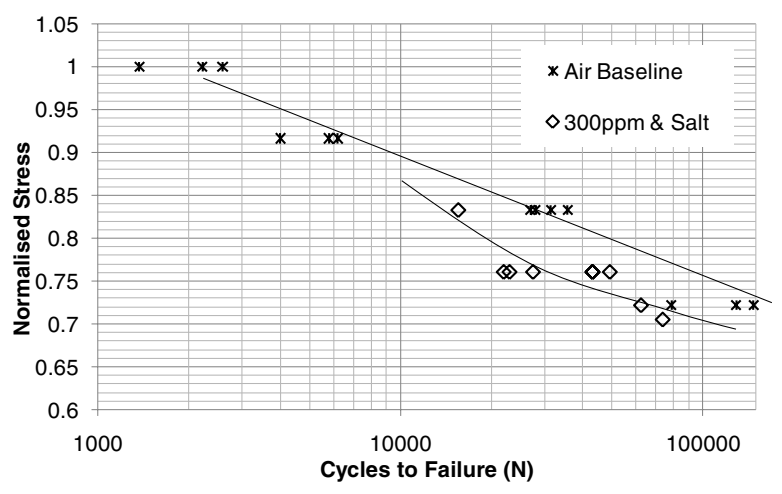

Figure 9. The effect of stress on fatigue life.

In contrast at intermediate flux/ "correct flux" specimens exhibit early stage/ stress enhanced type II hot corrosion. Specimen fractographic analysis shows a much thinner (typically 5-10 $\mu \mathrm{m}$ ) dual oxide layer, consisting of nickel oxide and mixed oxide, but the sulphur rich band between oxide and substrate is lacking. In this instance, sulphur is present as isolated titanium and chromium rich sulphide particles situated on the grain boundaries of the alloy ahead of the advancing oxide front, Fig. 8.

Localised breakdown of the oxide and cracking allow the formation of a localised defect or pit. This true "corrosion fatigue" process creates a highly localised stress enhancement feature and a significant reduction in life of up to $50-80 \%$ is seen.

\subsection{Effect of stress}

The effect of stress on fatigue life is shown in Fig. 9 and is plotted as peak stress versus number of cycles to failure. Comparing the data obtained at the single stress level, a consistent life and consistent mechanism with minimal scatter is observed.

A comparison between cylindrical specimen and notch specimen design indicates that the notch specimens exhibit a slightly longer life. Scatter is again minimal and the correct mechanism is observed. The slightly longer life that is seen in the notch specimens is therefore presumably due to critical stressed volumes and cracking into regions of significantly lower bulk stress.

Examining the data obtained at different peak stress, it is observed that in general, all specimens have experienced a reduction in life, although specimens tested at intermediate stress levels suffer the largest effect. Again, the data may be split into 3 separate regimes. At the highest

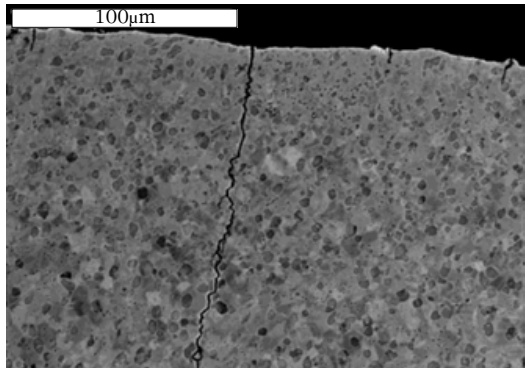

Figure 10. Example of cracking exhibited at high stress. Backscattered electron image of polished surface (un-etched) principal stress axis parallel to oxide layer.
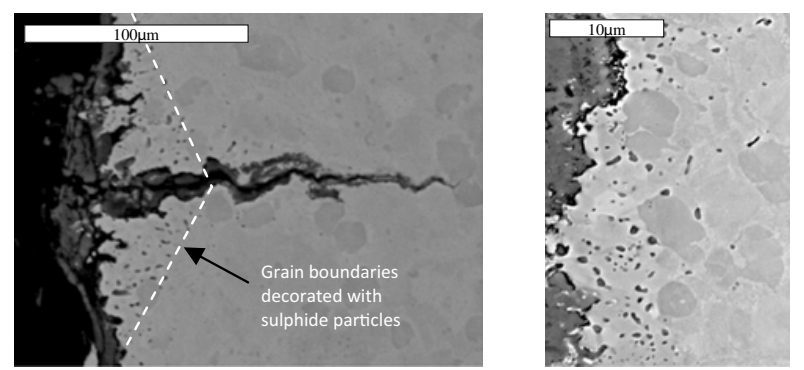

Figure 11. (a) Example of cracking at intermediate stress. Backscattered electron image of polished surface (un-etched) principal stress axis parallel to oxide layer, (b) high magnification micrograph of sulphide particle distribution.

stress level, the fatigue/or stress cycle is dominating the mechanism. There is very little corrosion evident on the specimens, (Fig. 10) no localised pitting, and consequently minimal reduction in fatigue life.

As the stress level reduces, corrosion mechanisms begin to play a role and start influencing crack initiation mechanisms, Fig. 11. In this instance, stress enhanced type II hot corrosion occurs whereby the characteristic dual oxide and sulphide decoration of grain boundaries is present.

Closer analysis surrounding early cracking on the gauge reveals a "V" shaped region of deeper grain boundary sulphides are present.

This would indicate that early cracking has taken place followed by a period of incubation where the crack struggles to grow, allowing diffusion of sulphur into the material down the crack faces. After some subsequent number of cycles, cracking then advances through the remaining section of the material from the base of the decorated "V". This initial cracking and incubation period appears related to pre-test surface treatment/peening processes.

As test stress drops further still a characteristic pit / $\mathrm{V}$ shape feature is generated at the initiation site, Fig. 12. In this instance, grain boundaries that have previously been decorated by sulphides have dropped out, creating a small stress raising feature. Again the size and shape of this feature suggests early cracking and incubation, but in this instance grain dropout is necessary to trigger final cracking presumably due to the lower stresses on the test specimen. More recent evidence suggests that if test stress is dropped further still, there is then insufficient stress for 


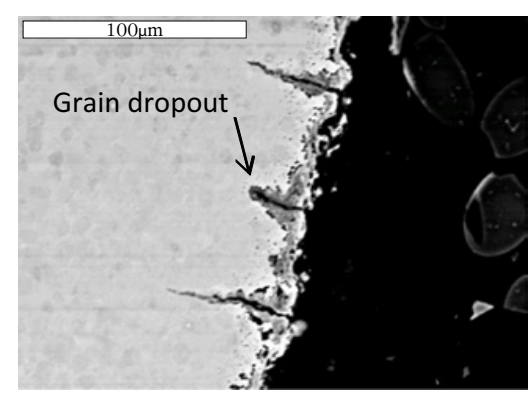

Figure 12. Example of cracking at low stress. Backscattered electron image of polished surface (un-etched) - principal stress axis parallel to oxide layer.

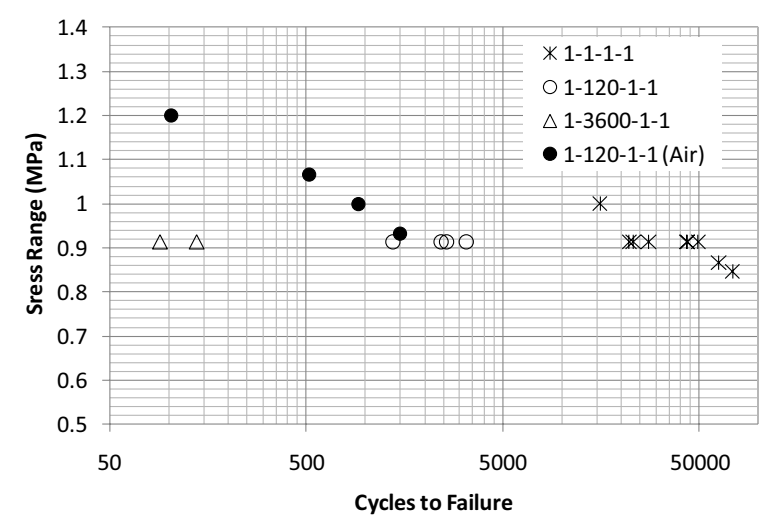

Figure 13. The effect of waveform on fatigue life.

grain dropout and number of cycles tends towards air $\mathrm{SO}_{x}$ only data.

Understanding of the influence of stress level and interactions with any peening treatment and local microstructure is key to fully understanding early cracking in these materials and is a subject of ongoing research [5]. Previous work [6] has shown that provided damage remains within the peened layer then cracking may be postponed. Whilst this work was performed utilizing prepitted specimens, so that the exact mechanisms of cracking were not investigated, it provided insight into the peening process itself and demonstrated that residual stress was not the dominant feature but the resulting deformed grains at the surface of the material that prevents cracking. This has subsequently been confirmed [7] whereby it was shown that by optimisation of this layer, it is possible to delay cracking under conditions of both corrosion and fatigue.

\subsection{Effect of waveform}

The effect of waveform on fatigue life is shown in Fig. 13 as peak stress versus number of cycles to failure. The data indicate that as the dwell at peak stress is increase, a reduction in life is observed.

This is characteristic of the materials behaviour under elevated temperature in air due to interactions with time dependent processes such as oxidation and creep. Comparing the results with baseline air data, it is noticed that whilst a reduction in life is observed under
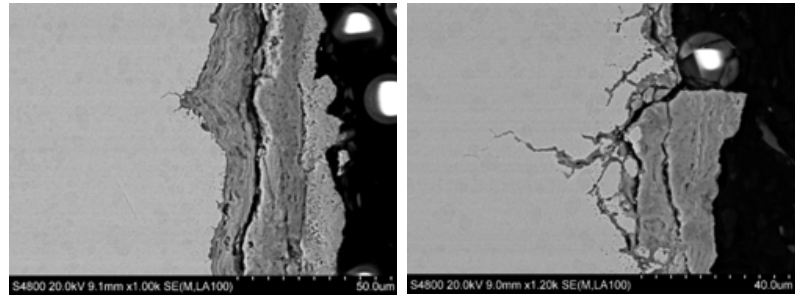

Figure 14. Oxide layer developed under 1 hour dwell conditions. Backscattered electron image of polished surface (un-etched) principal stress axis parallel to oxide layer.
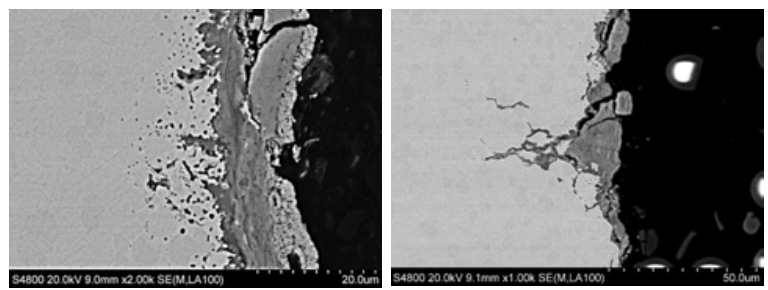

Figure 15. Oxide layer developed under 120 second dwell conditions. Backscattered electron image of polished surface (unetched) - principal stress axis parallel to oxide layer.

cyclic conditions, under conditions of 1-120-1-1 second conditions, the life compared with air is either similar or longer. No data is available for the 1 hour dwell under air for comparison. This again illustrates the intimate relationship between stress and environment. The absence of a reduction in fatigue life under extended dwell may be related to the absence of time independent processes and its relationship to oxide rupture. Detailed fractography reveals that under 1 hour dwell conditions a significant multi-layer oxide has developed with little evidence of sulphide decoration on grain boundaries, Fig. 14. In this case the sulphur is caught up in the advancing oxide front as a series of bands.

The formation of this stable oxide prevents diffusion of sulphur into the base material locally and thus prevents the formation of localised features. Examining sections taken from the 120 second dwell cycle, some evidence of localised breakdown of the oxide is present, Fig. 15. Where this has occurred, there are now sulphide particles decorating boundaries.

However, oxide/corrosion deposits are still significant indicating the development of a relatively stable layer that again provides protection to the base alloy. Whilst it is clear that the time dependent processes are dominating failure of the alloy at extended periods at peak stress, it is concluded that creep mechanisms are most likely influencing failure, rather than any corrosion fatigue mechanism. Evidence suggests that time independent processes are required to provide regular disturbances to the developing oxide that allows diffusion of sulphur to the base metal. Oxide film rupture mechanisms have been shown previously [7] to affect the fatigue performance of materials under corrosive conditions. In this case temperatures were insufficient to induce creep, however it was shown that the inclusion of dwell and rate of stressing, 
greatly affected life and under certain conditions life was extended beyond that in air.

\section{Conclusions}

The current paper has examined the influence of salt flux, peak stress and dwell on the low cycle fatigue performance of the nickel alloy 720Li. There is an intimate relationship between local salt level (flux), stress level and stress state, i.e. static or cyclic. The interaction with these variables with the work hardened layer present on the surface of all tested specimens as a result of the peening process directly affects the crack initiation process. If specific conditions of environment and stress are achieved an $80 \%$ reduction in fatigue life is observed.

The financial support of the Engineering and Physical Sciences Research Council through Grant No. EP/H500383/1 is gratefully acknowledged together with financial support from Rolls-Royce plc. The authors would also like to thank Dr Mark Hardy (RollsRoyce plc), Dr Daniel Child (Rolls-Royce plc), and Dr Simon Gray (Cranfield University) for technical help and support during the project.

\section{References}

[1] A. Encinas-Oropesa, G.L. Drew, M.C. Hardy, A.J. Leggett, J.R. Nicholls and N.J. Simms, Superalloys 2008, Edited by R.C. Reed et al, TMS (The Minerals, Metals \& Materials Society), Warrendale, PA, USA, 2008, pp 609-618

[2] W. Harrison, M. Whittaker, S. Williams, Materials 201, 6, 1118-1137

[3] H. Rosier, K. Perkins, S. Gray, Corrosion Science, (to be published)

[4] J. Sumner, A. Encinas-Oropesa, N.J. Simms and J.R. Nicholls, Oxidations of Metals, 80 (5-6), (2013), pp 553-563

[5] EPSRC/Rolls-Royce Strategic Partnership in Structural Metallic systems for Gas turbines, EP/H500383/1

[6] A. Encinas-Oropesa, N.J. Simms and J.R. Nicholls, Report ETC/0082, Cranfield University, UK, (2007)

[7] G.J. Gibson, K.M. Perkins, S. Gray and A.J. Leggett, Corrosion Science, (to be published)

[8] K.M. Perkins and M.R. Bache, International journal of Fatigue, 27, (2005), pp 1499-1508 